\title{
ROTATIONAL MOMENT SHAPE FEATURE EXTRACTION AND DECISION TREE BASED DISCRIMINATION OF MILD COGNITIVE IMPAIRMENT CONDITIONS USING MR IMAGE PROCESSING
}

\author{
$\underline{\text { Ravi Dadsena }}^{1}$, Deboleena Sadukhan ${ }^{1}$ and Ramakrishnan Swaminathan ${ }^{1}$ \\ ${ }^{1}$ Department of Applied Mechanics, Indian Institute of Technology Madras, Chennai, India
}

Corresponding Author: Ravi Dadsena

Email: ravidadsena34@gmail.com

https://doi.org/10.34107/BiomedSciInstrum.57.04228

\begin{abstract}
Mild Cognitive Impairment (MCI) is the preclinical, asymptomatic stage for Alzheimer's condition, which affects a large amount of the aging population around the world. Detection of MCI condition can ensure timely intervention needed for handling the disease severity. Morphological alterations of the Lateral Ventricle (LV) are considered a significant biomarker for diagnosing MCI conditions. This work aims at analyzing the shape alterations of LV from brain Magnetic Resonance (MR) images using Rotational moment shape features and differentiating MCI conditions using Decision Tree (DT) based classification. Trans-axial brain MR images are obtained from a publicly available OASIS database. Segmentation of LV is performed using the Reaction Diffusion level set, and the results are validated against Ground Truth. Rotational moment shape features are extracted from the segmented LV images. DT is implemented for the differentiation of control and MCI subjects. Results show that Rotational moment shape features are able to capture the alterations of LV in control and MCI subjects $(\mathrm{p}<0.05)$. The classification model achieves a high detection accuracy of $96.73 \%$ and an F-measure of $96.82 \%$. Hence, the proposed method can be used as an automated diagnostic tool to predict and monitor the cognitive decline in MCI subjects and can aid in disease management.
\end{abstract}

Keywords: Alzheimer's disease, Magnetic Resonance Images, Mild Cognitive Impairment, Lateral Ventricle, Rotational moment shape descriptor.

\section{INTRODUCTION}

Mild cognitive impairment (MCI) is an intermediate stage between normal aging and early dementia and is becoming gradually recognized as a risk factor for Alzheimer's Disease (AD). It is characterized by deficits in memory, language and thinking skills. [1]. Precise identification of MCI subjects at higher risk of developing AD can promote timely treatment initiation and possible prevention of dementia [2]. While clinical examinations continue to be the standard diagnostic criterion for MCI subjects, supplementary tests based on imaging measures and biomarkers are suggested to enhance diagnosis [3, 4].

Magnetic Resonance Imaging (MRI) is extensively used for the diagnosis of MCI due to its high resolution and soft tissue contrast. MRI of the brain reflects the atrophy of the hippocampus, entorhinal cortex, precuneus, caudate nucleus, thalamus and other subcortical regions [5]. Variation in the shape and volume of these structures influence the shape and volume of the Lateral Ventricle (LV). LV are fluid-filled regions located in the center of the brain with surrounding structures of gray matter and white matter. Expansion of LV is considered as an essential image-biomarker for differentiation of MCI conditions. In literature, volume measures are reported to be ineffective for disease diagnosis due to vast overlap 